\title{
Massif du Mont-Blanc : identification d'une structure aquifère majeure
}

\author{
The Mont-Blanc Massif : identification of a water-bearning \\ important structure
}

par Jean-Christophe Maréchal*

GEOLEP, Ecole Polytechnique Fédérale de Lausanne

The hydrogeology of Alpine crystalline massifs is still strongly unknown. The knowledge of groundwater reserves is important as far as tourism is developing in these regions. The Mont-Blanc road tunnel allows us to observe the crystalline massif at a large depth. This underground work has crossed over, during the drilling, a major water-bearning structure, at the origin of large water inflows $(1000 \mathrm{lls})$. The large discharge rates now still present $(200 \mathrm{l} / \mathrm{s})$ indicate that the concerned tectonic accident is at the origin of an important and permanent water resource. Observations made on the concerned water inflows permit to determine the behaviour of this water-bearing structure in the middle of the Mont-Blanc Massif.

\section{INTRODUCTION}

Les ressources en eaux souterraines de montagne sont rares et d'autant plus précieuses que le tourisme ne cesse de se développer dans ces régions. L'hydrogéologie des roches cristallines dans ce type de contexte a été peu étudiée jusqu'à présent. De nombreuses incertitudes subsistent quant au fonctionnement des aquifères cristallins montagneux, qu'il s'agisse de la perméabilité des massifs, de la vitesse de circulation des eaux ou de l'importance des réserves en jeu. Le tunnel routier du Mont-Blanc, percé au début des années 1960, offre une belle opportunité d'observer directement le milieu souterrain au cœur du Massif du Mont-Blanc. La coupe hydrogéologique offerte ainsi aux scientifiques permet d'observer l'aquifère en profondeur.

Lors du percement du tunnel routier du Mont-Blanc, une série d'importantes venues d'eau localisées sur un tronçon de 600 mètres de longueur ont été rencontrées par l'ouvrage au cœur du granite [1]. De forts débits sont encore présents dans cette zone quarante ans plus tard [2]. Les investigations menées récemment permettent de préciser la nature et le comportement de cette structure aquifère majeure au sein du massif du Mont-Blanc, ainsi que les principales caractéristiques qui la différencient du reste du massif.

* Actuellement au BRGM, Service Eau
L'identification et la caractérisation d'une telle structure hydrogéologique revêtent de l'importance non seulement pour l'hydrogéologue dans la perspective de l'exploitation future des ressources montagneuses en eau souterraine, mais également pour l'ingénieur dans le cadre de la réalisation d'ouvrages souterrains à grande profondeur en milieu montagneux.

\section{II —CONTEXTE DES VENUES D'EAU}

Le massif du Mont-Blanc, comme le massif des AiguillesRouges, est un massif alpin à matériel hercynien et plus ancien. Constitué de terrains cristallins et cristallophylliens, il possède une forme allongée du SW au NE (fig. 1). Le tunnel, perpendiculaire à l'orientation du massif, traverse celuici sous les Aiguilles du Midi et de Toule : sa longueur totale est de 11600 mètres. De l'entrée française (Chamonix), le tunnel recoupe sur $3100 \mathrm{~m}$ une série de schistes cristallins (fig. 2). Il traverse ensuite la masse granitique centrale sur environ $6775 \mathrm{~m}$ avant de pénétrer dans des terrains mésozoïques essentiellement carbonatés $(1300 \mathrm{~m})$. Le contact entre le granite et la couverture mésozoïque est de type tectonique tandis que celui avec les schistes correspond à un métamorphisme de contact : passage progressif $(425 \mathrm{~m}) \mathrm{du}$ complexe schisto-cristallin au granite intrudé postérieure- 


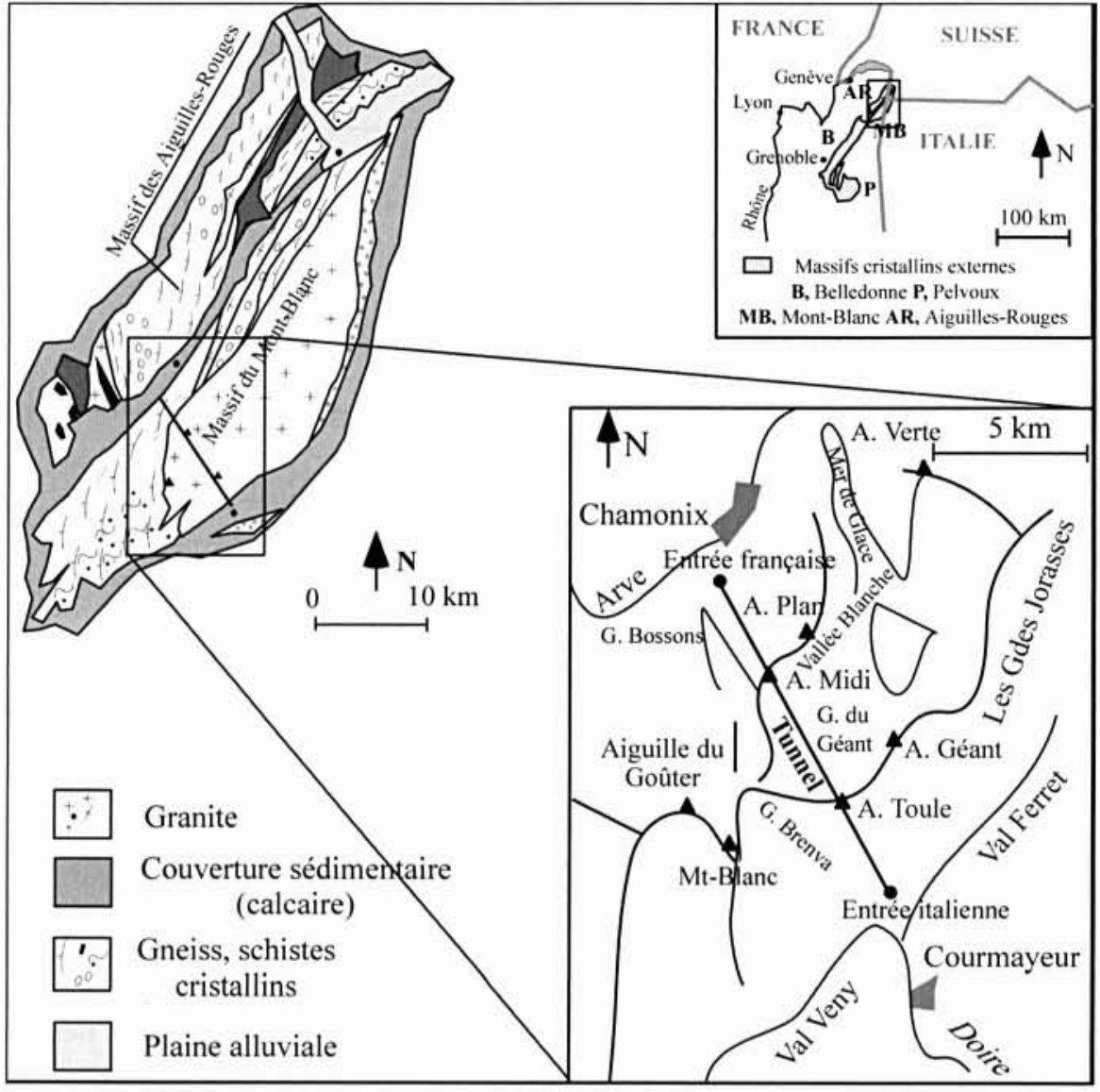

1. Carte géologique générale des Massifs Cristallins Externes du Mont-Blanc et des Aiguilles-Rouges (d'après [4]).

ment. Les formations sont affectées de fractures subverticales distribuées en éventail selon une direction parallèle à l'allongement du massif [3].

Les quantités d'eau enregistrées dans le tunnel routier du Mont-Blanc sont considérables : les jaugeages effectués à l'avancement ou juste après dérochement sont présentés sur la figure $2[5,6,1,7,8,9$ et 10$]$. Le débit total drainé par la galerie en cours d'avancement était de $1566 \mathrm{l} / \mathrm{s}$.

Les plus grosses venues d'eau sont localisées dans une zone fortement tectonisée, longue de 600 mètres, située dans la partie italienne du tunnel au cœur du granite, entre les PK $7,930-\mathrm{F}$ et $8,530-\mathrm{F}$ (PK-F : Point Kilométrique mesuré depuis l'entrée française). Elle correspond à un grand accident tectonique intra-massif dans lequel la fracturation s'intensifie. De grosses quantités d'eau y ont été rencontrées lors du percement avec des débits oscillant entre 12 et $800 \mathrm{l} / \mathrm{s}$ selon les venues situées entre les PK 7,932-F et 8,536-F, soit un total de $1084 \mathrm{l} / \mathrm{s}$ dès le percement. Les venues d'eau étaient telles qu'elles ont retardé d'un mois les travaux. Entre les PK 8,150-F et 8,530-F, [5, $6,1,7,8,10]$ et Gudefin (inédits) parlent d'une zone de cataclase et mylonitisation intenses, tout comme aux environs des PK 7,950-F et $8,060-\mathrm{F}$. Elle est directement suivie par une zone extrêmement peu fracturée. Dans le tunnel, cette dernière est totalement sèche - actuellement une seule venue (débit inférieur à $5 \mathrm{l} / \mathrm{min}$ ) est décelée entre les PK 8,523-F et 8,874-F [2]. Elle joue un rôle de barrière hydraulique favorisant le stockage d'eau et les écoulements dans la zone plus fracturée qui la précède. Ainsi les eaux circulant dans le massif se concentrent dans cette zone et s'écoulent vers le tunnel. En surface, une mylonite alpine d'orientation $\mathrm{N} 45^{\circ} \mathrm{E}$ a été observée, à proximité de la zone fortement tectonisée dans le tunnel [11].

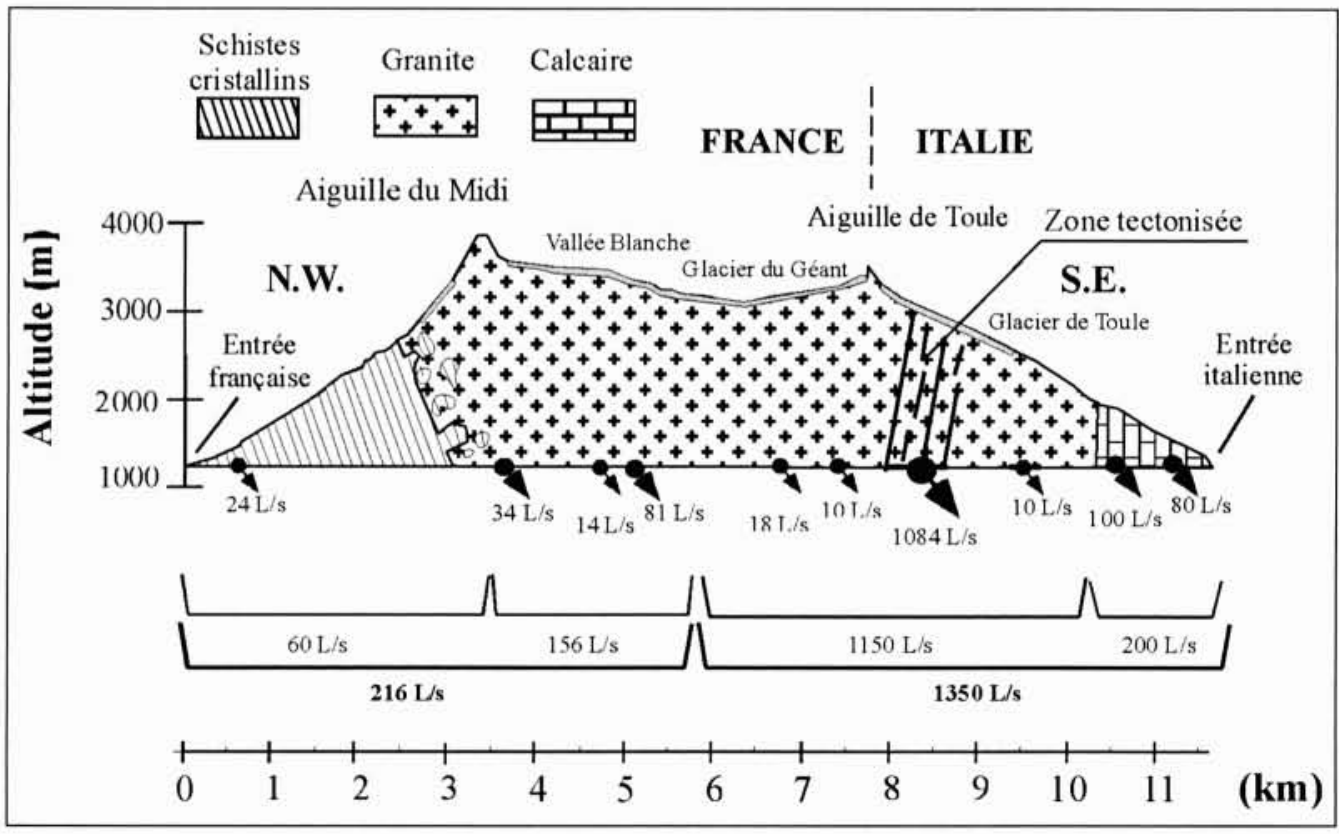

2. Venues d'eau enregistrées dans le tunnel du Mont-Blanc. Les débits ont été mesurés moins d'un jour après le percement. Une grosse venue est observable dans les granites aux environs du PK 8,0-F. 


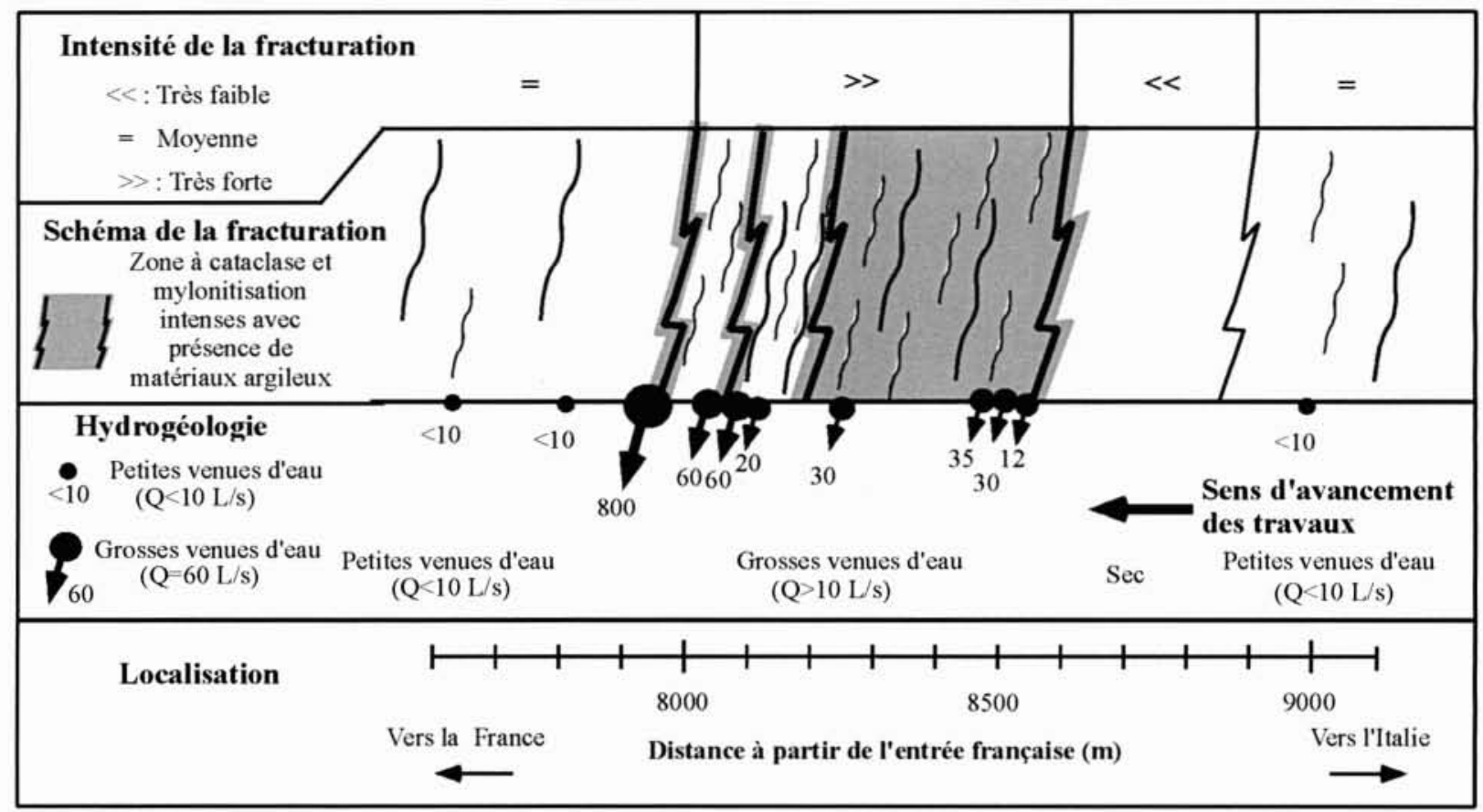

3. Fracturation et venues d'eau dans la zone fortement tectonisée du granite du Mont-Blanc.

La très forte venue enregistrée dans la partie italienne du tunnel a été précédée d'une série d'autres venues. Lors du percement, depuis l'attaque italienne, à l'intérieur de la zone tectonisée, les débits des venues d'eau ont augmenté avec l'avancement de 12 l/s au PK 8,536-F à 30 l/s au PK 8,516$\mathrm{F}$ à $60 \mathrm{l} / \mathrm{s}$ au $\mathrm{PK} 8,058-\mathrm{F}$ et $800 \mathrm{l} / \mathrm{s}$ pour la principale venue au PK 7,932-F (fig. 3). Ce phénomène tient de la nature de la zone drainante. Celle-ci est en effet constituée d'une succession de panneaux drainants et étanches selon la nature des matériaux la constituant. Les panneaux étanches (composés de matériaux argileux, produits extrêmes de la cataclase, observés dans cette zone) s'opposent aux circulations et favorisent la concentration des écoulements au contact de la kakirite avec le granite sain (exemple du PK 8,058-F). La superposition de panneaux aux propriétés hydrogéologiques différentes est à l'origine d'une anisotropie dans le tenseur de perméabilités de la zone: celle-ci possède une fonction drainante (verticalement, selon l'orientation des panneaux) et une fonction étanche (perpendiculairement à cette orientation). L'eau s'est alors accumulée derrière la zone et le percement du tunnel a provoqué la vidange du "château d'eau" ainsi constitué, ce qui explique l'intensité de la forte venue du PK 7,932-F.

\section{III — ANOMALIE GÉOTHERMIQUE}

Les profils de température des venues d'eau effectués dans le tunnel suivent globalement l'évolution de l'épaisseur de la

4. Profils de températures des venues d'eau dans le tunnel du Mont-Blanc.

Traits pleins : mesures effectuées dès le perc e $m$ e $n$ t (1959-1962). Cercles grisâtres : mesures effectuées en 1996.

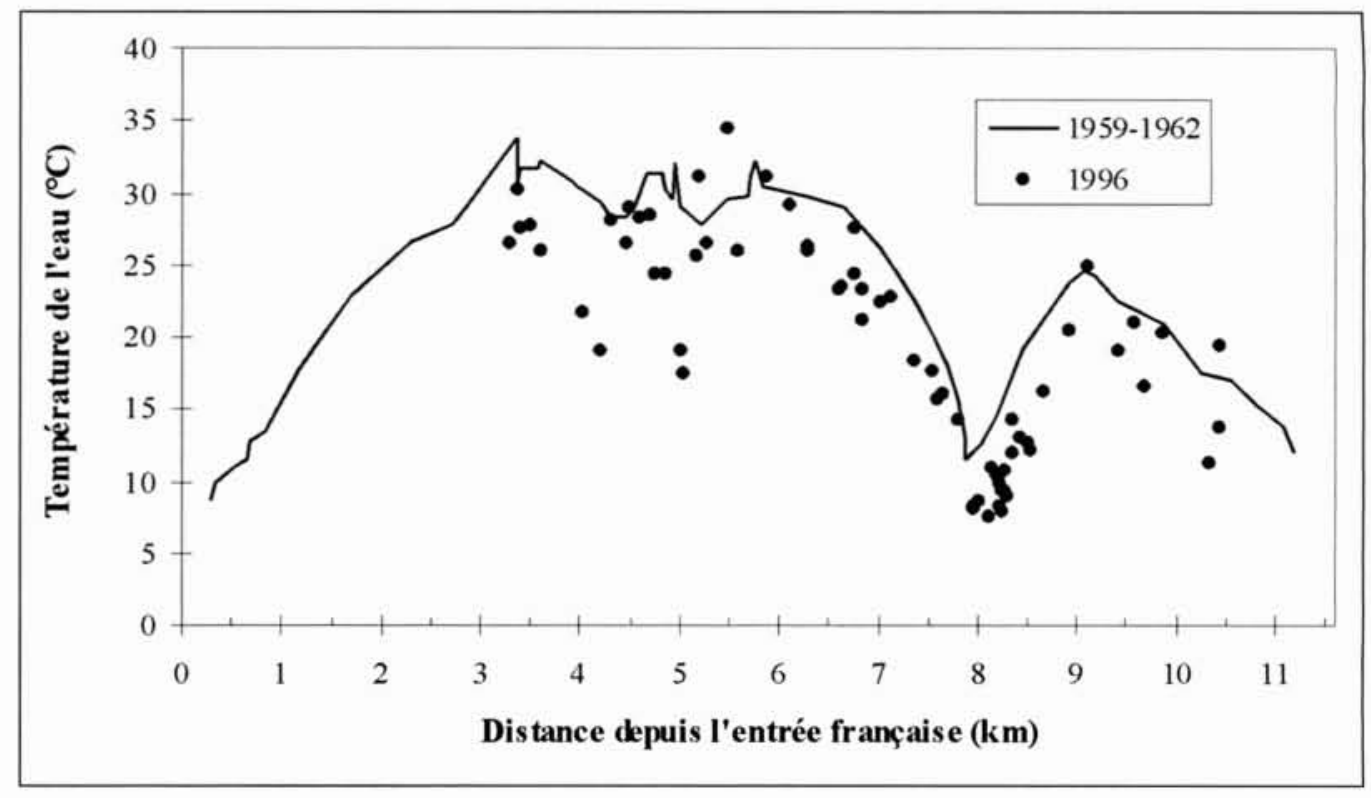




\begin{tabular}{|c|c|c|}
\hline $\begin{array}{c}\text { Zone } \\
{[\mathbf{P K}]}\end{array}$ & $\begin{array}{c}\text { Refroidissement } \\
\text { entre } \\
\text { 1961 } \text { et } \mathbf{1 9 9 6}^{{ }^{\circ} \mathrm{C} \text { C] }}\end{array}$ & $\begin{array}{c}\text { Vitesse } \\
\text { de refroidissement } \\
\text { [ }^{\circ} \text { C/année] }\end{array}$ \\
\hline 5,000 & 11 & 0,44 \\
8,200 & 4 & 0,16 \\
$3,000-7,000$ & 2 & 0,08 \\
\hline
\end{tabular}

Tableau 1. Evolution des températures des venues d'eau depuis le percement du tunnel.

Les venues sont caractérisées par un refroidissement variable selon leur position.

couverture rocheuse (fig. 4). Sur le profil réalisé dès le percement du tunnel (1959-1962), après une augmentation progressive des températures dans les schistes cristallins et les calcaires proches des deux extrémités, les eaux atteignent un palier de température au sein du granite central. Ce palier se situe aux environs de $30^{\circ} \mathrm{C}$, température relativement faible étant donné l'importante couverture rocheuse $(2300 \mathrm{~m})$. Le faible gradient géothermique $\left(=13^{\circ} \mathrm{C} / 1000 \mathrm{~m}\right)$ s'explique par le relief du massif (le gradient géothermique est plus faible sous les sommets que dans les vallées), la présence d'une couverture de glaciers et l'existence de circulations d'eau.

Une anomalie thermique négative est présente au droit de la zone à fortes venues (environs du PK 8,0-F). Elle était déjà bien marquée dès le percement du tunnel ; elle a joué un rôle précieux dans la galerie en réfrigérant celle-ci, permettant ainsi aux ouvriers de travailler dans des conditions acceptables [12]. Une autre petite anomalie apparaît au PK 5,000-F, liée à une autre venue d'eau.

Ces anomalies thermiques sont dues aux grosses quantités d'eaux froides (provenant de la fonte des glaciers) s'infiltrant en surface et s'écoulant vers le tunnel en refroidissant le massif à l'entoure. De précédentes analyses en oxygène18 sur les eaux du tunnel ont montré l'existence de circulations verticales entre les zones d'alimentation et le tunnel [13].

Dans la zone à grosses venues des PK 7,930-F à 8,530-F, les mesures de températures effectuées à l'avancement du tunnel (fig. 4) indiquent la préexistence de circulations d'eau dans cette zone avant le percement. En effet, les équipes italiennes ont constaté après $2 \mathrm{~km}$ de percement, un refroidissement subit des venues d'eau dans le tunnel alors que l'épaisseur de la couverture rocheuse continuait d'augmenter. Ces informations ont conduit les équipes de forage à se méfier de l'existence d'une zone à fortes circulations et ce à juste titre, puisque ces venues ont été rencontrées quelques mois plus tard et ont provoqué un arrêt des travaux pendant un mois [12]. Les circulations pourraient être orientées perpendiculairement à la galerie et ce, au sein de la zone fortement fracturée et tectonisée traversée par le tunnel. Celle-ci, d'orientation $\mathrm{N} 45^{\circ} \mathrm{E}$ parallèle aux grands accidents affectant le massif $[14,3]$, constitue un panneau plus perméable dans lequel les écoulements se concentraient avant le percement de la galerie. L'exutoire de ces circulations n'est pas formellement connu. Il peut s'agir probablement d'exutoires localisés dans la vallée glaciaire de la Brenva, située quelques centaines de mètres au sud du tunnel et parallèle à celui-ci.

$\mathrm{Si}$ le profil de température effectué récemment a une forme globalement similaire à celle du profil réalisé dans les instants qui ont suivi le creusement, on constate tout de même un refroidissement général des venues (fig. 4). Les venues chaudes se refroidissent assez peu en général : elles passent de $30^{\circ} \mathrm{C}$ en moyenne à $28^{\circ} \mathrm{C}$. Par contre, des chutes de température importantes se sont produites dans les deux zones différenciées précédemment (tabl. 1 ).

Les deux zones, sièges des deux principales venues d'eau, se sont refroidies assez sensiblement au cours des années. A l'opposé, le reste du massif (PK 3,000 - 7,000-F), moins perméable, subit un refroidissement moins important. Par ailleurs, le taux de refroidissement est directement relié à l'importance des circulations préexistant dans le massif avant le percement du tunnel. Ainsi le refroidissement est plus grand dans la zone perméable ne possédant pas ou peu d'exutoire avant le percement du tunnel : il s'agit de la zone $5,000-\mathrm{F}$, située plus à l'intérieur du massif, soit loin des exutoires potentiels. Elle possède le refroidissement le plus important de tout le tunnel. La zone du PK 8,200-F étant le siège de circulations avant le percement du tunnel, subit un refroidissement plus faible que la zone $5,000-\mathrm{F}$ mais cependant plus important que la zone centrale du granite.

Les observations effectuées au cours des précédentes études sur les eaux du tunnel $[1,13,15,16]$ permettent de préciser quelque peu l'évolution des températures depuis la réalisation de l'ouvrage (fig.5). Les venues chaudes des PK $3,500-\mathrm{F}$ et $4,500-\mathrm{F}$ ont évolué dans un premier temps avec un refroidissement de $2^{\circ} \mathrm{C}$ en près de 17 ans. Ensuite, elles ont atteint un plancher et semblent ne plus évoluer. Considérées comme représentatives du comportement général des venues chaudes, elles sont maintenant caractérisées par un état apparemment thermiquement permanent. La venue du PK 8,200-F (une des venues froides de la zone tectonisée) montre une évolution parfaitement linéaire depuis la construction du tunnel. Thermiquement, elle n'est pas encore en équilibre : son état est transitoire, elle se refroidit encore à 1'heure actuelle. $\mathrm{La}$ venue froide du PK 5,000-F se comporte de manière intermédiaire puisqu'il semble qu'elle se soit refroidie rapidement au début suite à la mise en circulation des eaux, et évolue plus ou moins linéairement actuellement. L'évolution des températures traduit donc assez fidèlement l'influence du tunnel sur les circulations d'eau. Une modélisation mathématique des écoulements et échanges de chaleur dans le massif à très long terme $[2,17]$ montre que c'est l'ensemble des venues d'eau dans le tunnel qui sont dans une phase de refroidissement depuis la fin de la dernière période glaciaire (moment où les infiltrations d'eaux froides

5. Evolution dans le temps de la température des venues d'eau dans le tunne du Mont-Blanc. 


\section{クécanique des fluides et applications}

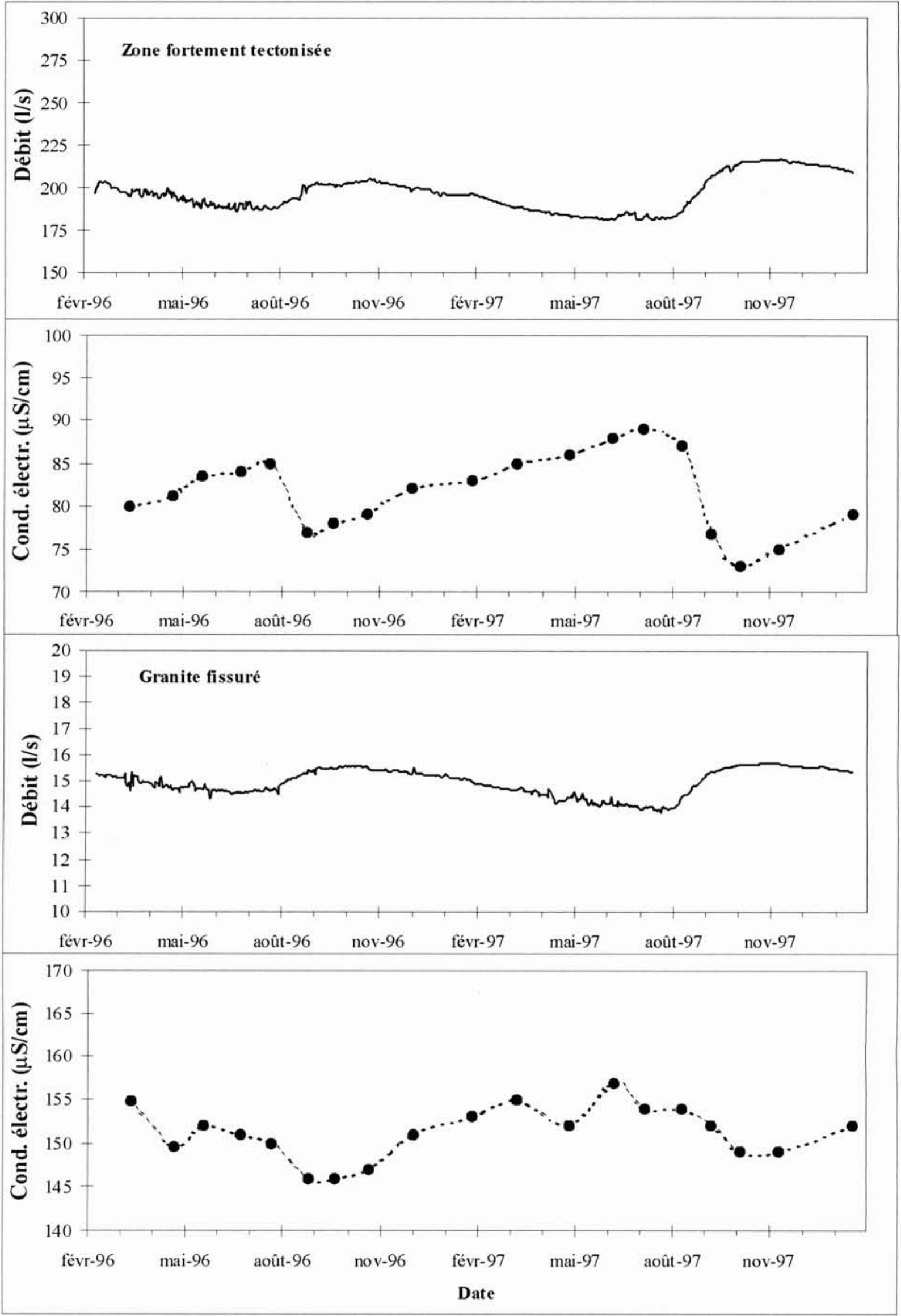

6. Evolution des débits et conductivités électriques des eaux du collecteur principal. 